\title{
Research at Brown Boveri
}

\section{H. R. Zeller, Baden}

(Research Centre)

The present position of Brown Boveri as an internationally competitive industrial enterprise rests to a large extent on the determination and ability of management to create a foundation through research and development which will support maximum technical achievement. Technological competence, however, is not something that can be maintained without effort. Only those who continually strive to expand knowledge through intensive research and development will remain in the forefront. Over 5000 employees are engaged in the area of research and development, the support for which amounts to $8 \%$ of the Group's annual turnover. Brown Boveri have put into effect a concept of research and development on three levels adapted to the geographically decentralized structure of this multinational company.

The Testing, Development and Design Departments of the Divisions constitute the first level. The Divisions are the line units of the three large Companies of the Group in Germany, Switzerland and France and are managed as profit centres. Because of this structure, proximity to market and customers is assured and product development and improvement can be carried out quickly and effectively by meeting demands for economy, quality and reliability.

The second level consists of the Central Laboratories of the national companies. Means have been placed at their disposal to support the efforts of the development departments in fundamental aspects relating to materials sciences, chemistry, measurement techniques and other areas.

Corporate Research is the third level and reports directly to the Managing Committees. It may be characterized by its working according to the methodology of the basic sciences, the medium to long-term orientation of its projects and its organization according to scientific disciplines, each of which can simultaneously influence several different product areas.

The Corporate Research at Brown Boveri was founded as a result of a need which developed during the 1960's. The company's international position, chiefly as a supplier of energy generation and transmission systems, requires not only a strong and determined development effort but also a steady flow of research results and a close contact with the scientific community. Today a large part of Brown Boveri's corporate research is located at Dättwil near the company's headquarters in Baden (Switzerland) and smaller fractions in Heidelberg (Germany) and Le Bourget (France).

After the first ten years have passed it is fair to ask the question whether Brown Boveri's corporate research has been able to establish a strong position within the company and within the scientific community; i. e., whether for the company it has become a key contributor to technical progress and whether for the scientific world it is now an institution which not only draws on the general scientific pool of knowledge but also contributes to it. Instead of trying to answer these questions by citing a complete list of former and present research activities, we shall in this paper concentrate on two projects which have at the same time strong technical and scientific aspects.

\section{Death of a Switching Arc}

Energy research in plasma physics consists not only in designing ever larger fusion devices but also in trying to understand, for instance, the physical phenomena in a power circuit breaker.

Until very recently the design of a circuit breaker was more an art than a science and required years of experience followed by prolonged testing, which at the voltage and current levels involved is extremely expensive.

Our research efforts started with the study of a well defined model system, the axially blown arc. Using the most advanced experimental tools of modern plasma diagnostics, together with analytical and computer modelling methods it was possible to identify, study and understand the mechanisms which control the switching properties.

It is not the arc directly but the extinction of the arc which is of interest in a circuit breaker. Obviously the critical stage for extinction is when the current passes through zero in the $A C$ cycle. Our experimental and theoretical work has demonstrated that at current zero, the plasma consists of an extremely thin arc channel. The shear flow near the arc boundary produces strong turbulence, a mechanism which rapidly dissipates heat,
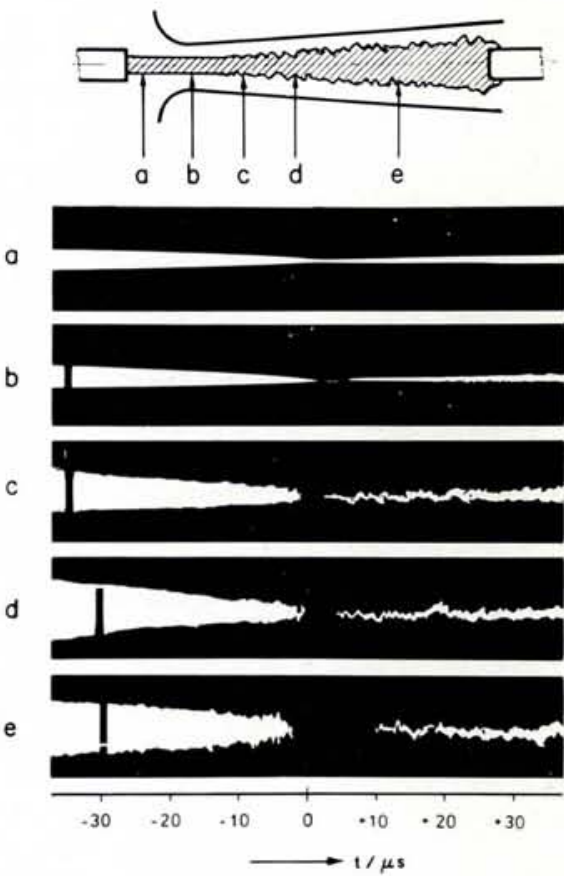

Fig. 1 - Extinction and reignition of a plasma arc in an axial ultrasonic flow. The Schlieren photographs show the time development of the arc at different points in the nozzle. $t=0$ denotes current zero in the arc cycle. Upstream of the nozzle constriction the flow is laminar, downstream it is turbulent. 
cools the arc channel and causes current interruption.

The further cooling is governed by a rather slowly decaying channel of hot gas. It is this decay which determines whether the circuit breaker can withstand the rapid voltage rise or whether the arc will fail dielectrically. The dielectric breakdown in cold gases is a well understood phenomenon. In hot gas, however, the recovery of electric strength depends on the electric charge carrier generation and destruction mechanisms which are related to the thermodynamic and kinetic properties of the electrons and ions in the hot gas and to the applied electric field. All these interacting effects have to be studied with a time resolution in the microsecond to millisecond range.

The basic investigation of the phenomena in the arc at, and shortly after, current interruption has led to improved circuit breakers and to a detailed understanding of the ultimate limits of this switching process as a function of the material parameters. This is an important step towards replacing the empirical approach by modern methods of quantitative predictive engineering.

\section{Superionic Conductors}

A major drawback of electricity is that it cannot be stored without conversion to another energy form. Electrochemical storage in the form of batteries is one solution to this problem.

A large scale high power application requires batteries with light, reactive, cheap and abundant reactants resulting in an economic high energy and power density storage. These conditions are met by the Na-S battery. In this battery, the liquid reactants sodium and sulphur are separated by a solid state $\mathrm{Na}^{+}$conductor. The Na-S battery has an energy density several times larger than standard lead-acid batteries. Major future applications

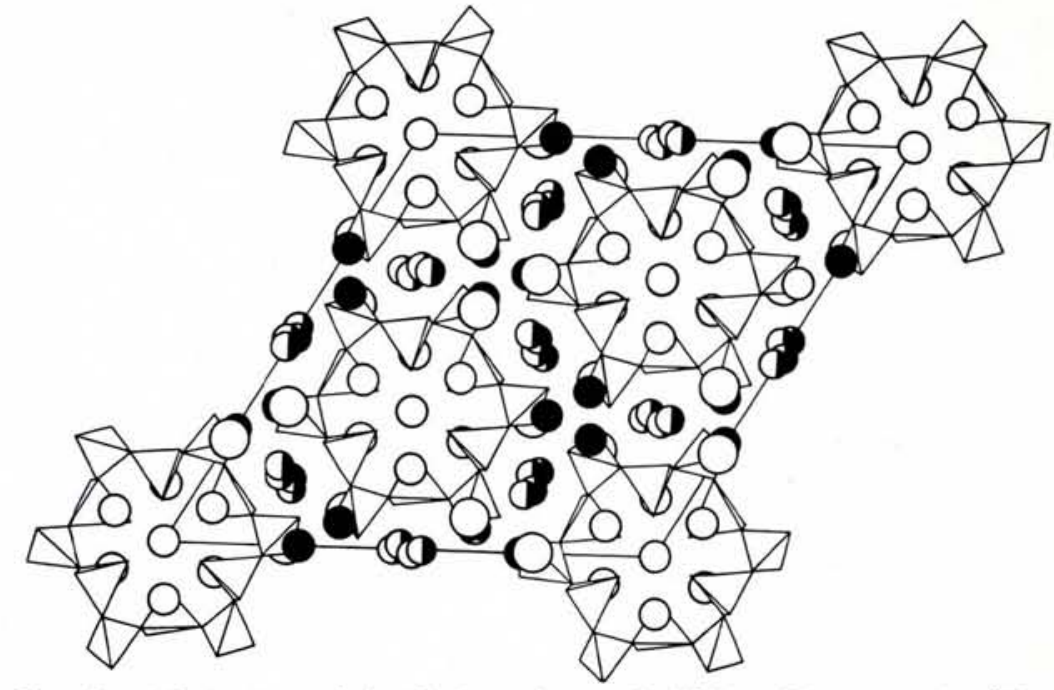

Fig. 2 - Crystal structure of the $\mathrm{Na}^{+}$conductor $\mathrm{Na}_{5} \mathrm{YSi}_{4} \mathrm{O}_{12}$. Shown are the $\mathrm{SiO}_{4}$ tetrahedra, $\mathrm{Y}$ sites (large circles) and $\mathrm{Na}$ sites (small circles). Only the $\mathrm{Na}^{+}$outside the silicate rings are mobile. Their average site occupation is less than one and is schematically shown (black circles $=100 \%$ occupancy, white circles $=0 \%$ occupancy). areas are envisaged in the field of traction and peak load levelling in electrical networks.

Such battery systems are under active development in many laboratories, also at the Brown Boveri research laboratory in Heidelberg which belongs to the leading groups in this field.

The heart of the Na-S battery and of other competing battery concepts is a solid electrolyte or superionic conductor. A superionic conductor is characterized as being a solid with an ionic conductivity approaching that of a liquid. The $\alpha$-phase of Agl for instance has an $\mathrm{Ag}^{+}$conductivity $>1(\Omega \mathrm{cm})^{-1}$ or more than ten orders of magnitude bigger than a standard solid and greater than concentrated sulphuric acid.

The phenomenon by itself has been known since the 30's but only recently has the field attracted a broad interest. As the chemical, structural and physical conditions for the occurrence of this remarkable phenomenon have become better understood, many new materials have been found which

\section{INTERNATIONAL ADVANCED STUDY INSTITUTE ON}

THEORETICAL ASPECTS AND NEW DEVELOPMENTS IN MAGNETO-OPTICS

16 July - 28 July, 1979

University of Antwerpen (Rijksuniversitair Centrum) BELGIUM

Organizing committee: J.T. DEVREESE (Chairman), L. F. LEMMENS. V. E. VAN DOREN.

Mailing address for application and information: Prof. Dr. J. Devreese, NATO Advanced Study Institute, State University of Antwerpen (R.U.C.A.), Groenenborgerlaan 171, B - 2020 ANTWERPEN (Belgium).

Deadline for registration: 15 June, 1979. exhibit superionic conductivity. For instance a class of $\mathrm{Na}^{+}$conductors of the type $\mathrm{Na}_{5} \mathrm{ReSi}_{4} \mathrm{O}_{12}$ ( $\mathrm{Re}=$ Rare Earth) has been independently discovered by Du Pont, MIT and Brown Boveri.

From a scientific point of view, superionic conductors are extremely complex systems. A necessary condition for high ionic conductivity is a disordered sub-lattice of the conducting ions with more than one site per ion. Since diffusion is the ultimate anharmonic event, at least some of the lattice modes are strongly anharmonic. One thus has to treat diffusion of a concentrated system of strongly interacting particles driven by the dynamics of an anharmonic and disordered lattice.

Recently a formalism was developed at Brown Boveri which allows a microscopic treatment of diffusion in such systems. It is found that in the best conductors, the coupling between diffusive and oscillatory motion has pronounced effects on the conductivity. The formalism also makes it possible to evaluate the approximations in simpler models and to interpret the parameters of phenomenological models microscopically. This is not only of academic interest but also an important step towards a more effective search for new materials.

These two examples of industrial research projects are intended to illustrate that the quality criteria for industrial research are not much different from those of academic research. In both cases, success requires new and original ideas, a mastering of scientific methods and the ability to put one's own work into perspective with scientific and technical progress in general. 